\title{
Cure of Vesico-Vaginal Fistula by Abdominal Section.
}

\author{
By E. Hastings Tweedy, F.R.C.P.I. \\ Gynacologist, Steevens' Hospital, Dublin.
}

THE technique for the repair of vesico-vaginal fistula, though greatly improved within recent years, still leaves much to be desired. It would be an interesting subject for the statistician to determine the number of cures resulting from the first operation. For my own part I confess to failure in a large number of cases, but, save in one instance, $I$ have never failed to bring about ultimate cure.

The following history not only shows the difficulties that beset an operator but also demonstrates how extensive are the procedures that have sometimes to be faced.

E.D., 46 years, admitted to the Rotunda Hospital August, 1910. Had a "Wertheim" hysterectomy performed for squamous-celled carcinoma of the cervix. Two days after operation she developed a vesico-vaginal fistula. Four weeks later an attempt to close this through the vagina utterly failed, the bladder being fixed so high in the pelvis that it would not pull down enough to give access to the fistula. The patient refused further treatment and was discharged, to return on October 24 because of the discomfort from continuous dribbling of urine.

Examination revealed a fistula $1 \times 1 \frac{1}{2} \mathrm{in}$. in the vault of the vagina through which part of the bladder wall had prolapsed. From the vagina it was absolutely impossible to reach or close this fistula. On October 28 the lower half of the old scar was cut away and the abdomen opened. There were no adhesions or evidence of malignant disease. An assistant's finger was passed into the vagina and an incision made on it. The back wall of the bladder was opened and the incision was continued down until it communicated with the fistula. The edges of the latter were freshened. The bladder and vaginal openings were now continuous. Beginning behind, the vagina was closed with interrupted catgut sutures down to the level of the fistula. The fistula and incision in the bladder were closed 
with interrupted catgut sutures, starting at the anterior extremity and working backwards. Finally the peritoneum was closed over the whole raw surface with interrupted catgut. A gauze plug was placed along the peritoneal wound and the end brought out the lower angle of the abdominal incision. The abdomen was closed in layers. A wing catheter was left in for 11 days. There was never any leakage.

Discharged November 19, 1910, completely cured. 